\title{
A vueltas con el gnomon. Buscando soluciones a problemas
}

\section{On the gnomon. Searching answers to problems}

\author{
Valentín Gavidia \\ Dpto. Didáctica Ciencias Experimentales y Sociales \\ Universidad de Valencia \\ Valentin.Gavidia@uv.es
}

RESUMEN - Son frecuentes las aproximaciones cualitativas e intuitivas que se hacen en la escuela sobre el movimiento aparente del Sol por el firmamento que tratan de proporcionar al alumnado observaciones para que construya un modelo del sistema Sol-Tierra. El presente trabajo muestra la forma de obtener datos con mayor exactitud de manera que faciliten al alumnado una visión más amplia y profunda de dicho modelo, que lo capacite para enfrentarse y resolver problemas cotidianos y los pueda aplicar a diferentes contextos, como el cuestionamiento de situaciones sociales en las que interviene la luz solar.

PALABRAS CLAVE: astronomía; gnomon; trayectoria del Sol; modelo Sol-Tierra; problemas científicos; ideas previas.

\begin{abstract}
There are common the intuitive and qualitative approaches made in the School on the apparent movement of the sun across the sky in order to provide students observations to construct a model of the Sun-Earth system. This paper shows how to obtain data more accurately to provide students with a broader and deeper view of the model, to enable them to confront and solve everyday problems, and can be applied to different contexts, such as questioning social situations involving the sunlight.
\end{abstract}

KEYWORDS: astronomy; gnomon; path of the Sun; Sun-Earth model; scientific problems; previous ideas. 


\section{INTRODUCCIÓN}

La Astronomía diurna, y en concreto la trayectoria del Sol por el firmamento, es uno de los temas estudiados en la escuela, tanto en la educación primaria como en la secundaria. Permite introducir al alumnado en el rigor de las observaciones, en la presentación de problemas, en la argumentación (López Gay et al., 2009; R. Ros, 2009). Posibilita utilizar la Historia de la ciencia para atender el cambio conceptual que esperamos en el alumnado, y presentar las diferentes visiones del mundo y de la ciencia. Es un campo en el que se han estudiado las ideas previas de alumnos y profesores (Klein, 1982; Jones et al., 1987; Camino, 1995; Afonso et al, 1995; De Manuel, 1995; Baxter, 1989; A. Vega, 2001; etc.) y se han elaborado programaciones didácticas que tratan de recoger las preguntas y los problemas más frecuentes que nos hacemos al mirar al cielo (Domenech et al. 1985; Domenech y Bella, 2000; García Barros et al., 1996; Moreno, 1997; Navarrete, 1998; A. Gangui, M. Iglesias y C. Quintero, 2009). En definitiva, es un tema apasionante al que el profesorado le dedica tiempo y atención y en el que suele encontrar satisfacción por los resultados educativos que obtiene (Cardenete, 2011).

No obstante, diversas investigaciones han puesto de manifiesto las dificultades en el proceso de enseñanza-aprendizaje de la Astronomía (Bach y Franch, 2004; Solbes y Palomar, 2013) y en el hecho de que los obstáculos que han aparecido a lo largo de la Historia de la ciencia se vean reflejados en las dificultades que tiene el alumnado en la construcción de su conocimiento en esta temática, por lo que debemos tenerlos en cuenta en nuestra acción didáctica (Vosniadou, 1991). Baxter (1989) sugiere que para la comprensión de la astronomía más cotidiana debemos tener en cuenta cuatro dominios fundamentales: el planeta Tierra en el espacio, el día y la noche, las fases de la Luna, y las estaciones, todo ello dirigido a la construcción del modelo Sol-Tierra- Luna.

El presente artículo pretende presentar algunas aportaciones que faciliten la construcción de dicho modelo y, por tanto, comprender e interpretar adecuadamente los fenómenos astronómicos observables. Sin embargo, nos centraremos en el problema de las estaciones y sus causas para una interpretación correcta de los fenómenos cotidianos observables, dejando de lado los aspectos relativos a la Luna. Comenzamos trabajando con el alumnado de primer curso de educación secundaria obligatoria, puesto que la mayoría tiene interiorizado conceptos como la forma del Sol, la Luna y la Tierra (aunque no sepan ofrecer argumentos a partir de observaciones desde la propia Tierra y acudan a las imágenes de los satélites) y la causa del día y la noche (Bryce y Blown, 2013).

Nuestra propuesta consiste en pasar de las primeras actividades que se hacen en educación primaria, que suelen ser aproximaciones cualitativas e intuitivas que ayudan a formarse una idea del modelo Sol-Tierra, a realizar observaciones más continuadas y con mayor precisión que nos posibiliten realizar aproximaciones más exactas, las cuales nos permitirán profundizar en el modelo Sol-Tierra a lo largo de la educación secundaria. Terminaremos aplicando el modelo construido a diversos contextos abordando problemas y cuestiones de mayor trascendencia, tanto personal como social, que presentaremos al alumnado de $1 .^{\circ}$ de bachillerato, en la asignatura de Ciencias para el Mundo Contemporáneo, en la que existe un tema denominado El lugar de la Tierra en el Universo, y en el Máster de Profesor de Educación Secundaria.

\section{PRIMEROS PROBLEMAS E IDEAS PREVIAS}

Señalan Blown y Bryce (2010) que las ideas que tiene el alumnado sobre el Sol, la Tierra y la Luna, así como sus movimientos aparentes, están estrechamente relacionados y configuran una visión holística del cielo y la Tierra, formando parte de un súper concepto de Tierra. Ello nos da a entender que las actividades y experiencias didácticas que presentemos deben encaminarse a obtener esa visión global que significa la construcción del modelo que explique los fenómenos astronómicos observables, teniendo 
en cuenta que sobre la mayoría de ellos el alumnado ha ido dando una explicación alternativa pero convincente para ellos, lo que se traduce en una dificultad ańadida para una interpretación correcta.

Sobre la secuencia de las estaciones y la variación del frío y calor a lo largo de un año, Baxter (1989) detecta entre el alumnado de los primeros niveles varias ideas alternativas, como son que el frío se debe a que las nubes detienen el calor o a la participación de la vegetación en la temporada fría; pero la gran mayoría, de entre 15 y 16 años, señala que se debe a la proximidad o lejanía del Sol. Pocos lo explican en términos de la inclinación del eje y la perpendicularidad o inclinación de los rayos solares que llegan a la Tierra. Hay evidencias de que a medida que avanza la edad del alumnado se abandonan las ideas más simples e ingenuas, sin embargo existen concepciones verdaderamente "ancladas» que son de difícil superación (Baxter, 1989; Camino, 1995; Atwood y Atwood, 1997; Dove, 2002; Bryce y Blown, 2012) pues llegan a la edad adulta, e incluso se han observado entre el profesorado de varios lugares como Inglaterra, China o Nueva Zelanda.

Varias sugerencias se han expuesto para explicar por qué los estudiantes encuentran obstáculos al interpretar los acontecimientos astronómicos. Entre ellos se cita la conciliación de las experiencias cotidianas, como la observación de la salida y puesta del Sol, con los modelos abstractos que intentan explicar por qué ocurren estos fenómenos, así como la interpretación de los diagramas de dos dimensiones que intentan representar el espacio tridimensional, o el uso de terminología ambigua como dar vueltas, moverse alrededor de, girar, ángulo de rotación, inclinación del eje, etc. (Vosniadou, 1991; Parquer y Heywood, 1998; Dove, 2002) o la posible confusión entre el día de 24 horas y el día de horas de luz.

Este último autor indica en su trabajo que el 91\% del alumnado de 12 años es capaz de explicar las causas del día y de la noche seńalando la rotación de la Tierra sobre su eje. Una razón de este resultado puede ser debido al hecho de que en este aspecto solo intervienen dos elementos: la Tierra y el Sol, y una observación directa sencilla. Sin embargo, son muy pocos los alumnos que explican la razón de las estaciones, seguramente porque intervienen un mayor número de elementos: el movimiento de rotación, el de traslación por la órbita de la Tierra, y especialmente la posición de su eje de rotación en relación con el Sol. A ello hay que añadir que los datos necesarios para una interpretación correcta se obtienen de una observación más compleja y prolongada en el tiempo. Tanto entre el alumnado de 16 años como entre los estudiantes de Maestro de Educación Primaria, e incluso entre los Maestros en ejercicio, es bajo el porcentaje que señalan como causa de las estaciones la inclinación del eje de la Tierra con relación al Sol, y muy pocos hacen mención a la mayor o menor inclinación de los rayos solares. Ninguno trató de explicar por qué la duración del día cambiaba con las estaciones.

Parker y Heywood (1998) señalan la importancia de considerar esta variación del día y la noche a lo largo del año relacionándolo con la inclinación constante del eje de la Tierra con respecto al plano orbital para comprender la causa de las estaciones, y Klein y Riskin (2003) y Gangui, Iglesias y Quintero (2009) indican que es necesario una observación diaria de la altitud del Sol para comprobar cómo esta va cambiando a lo largo del ańo, al igual que ocurre con el lugar de la salida y la puesta del Sol. Plummer y Krajcik (2010) apuestan por ofrecer explicaciones a los fenómenos observados desde una perspectiva terrestre y por estudiar la ruta del Sol, que consiste en «un arco suave en el cielo que cambia lentamente de longitud y de altitud a través de las estaciones». Las distintas rutas se deben a la rotación de la Tierra y a su órbita alrededor del Sol sobre su eje inclinado, y está mediada por nuestro lugar de observación en la superficie curva de la Tierra. Señalan que es necesaria la adquisición del concepto de que «el Sol no pasa perpendicularmente sobre nuestras cabezas todos los días», como idea para entender que la ruta del Sol varía a medida que transcurren los días y hay que tener en cuenta nuestro punto de observación.

Atendiendo a todas estas observaciones y la forma en la que se han obtenido, que en general ha sido a través de entrevistas, cuestionarios, dibujos y diagramas realizados por los propios encuestados y co- 
mentarios sobre los propios dibujos, hemos querido conocer nuestro punto de partida en la secuencia de enseńanza-aprendizaje que emprendemos con el alumnado utilizando un instrumento diferente. Para ello, la primera actividad que realizamos para comenzar a construir el modelo Sol-Tierra es estudiar la sombra que proyectan los objetos y hacer el seguimiento de esta. Es lo que pretende la actividad en la que se pide dibujar con la mayor exactitud posible la sombra que arroja un árbol en tres momentos concretos del día, dejando libertad para determinar las variables necesarias para ello.

Los resultados que obtenemos en la mayoría de los casos con esta actividad, bien sea del alumnado de educación primaria, secundaria, magisterio, del Máster de Profesor de Secundaria, e incluso del propio profesorado son coincidentes con la bibliografía revisada; los resumimos de la manera siguiente y se pueden observar en la figura 1.

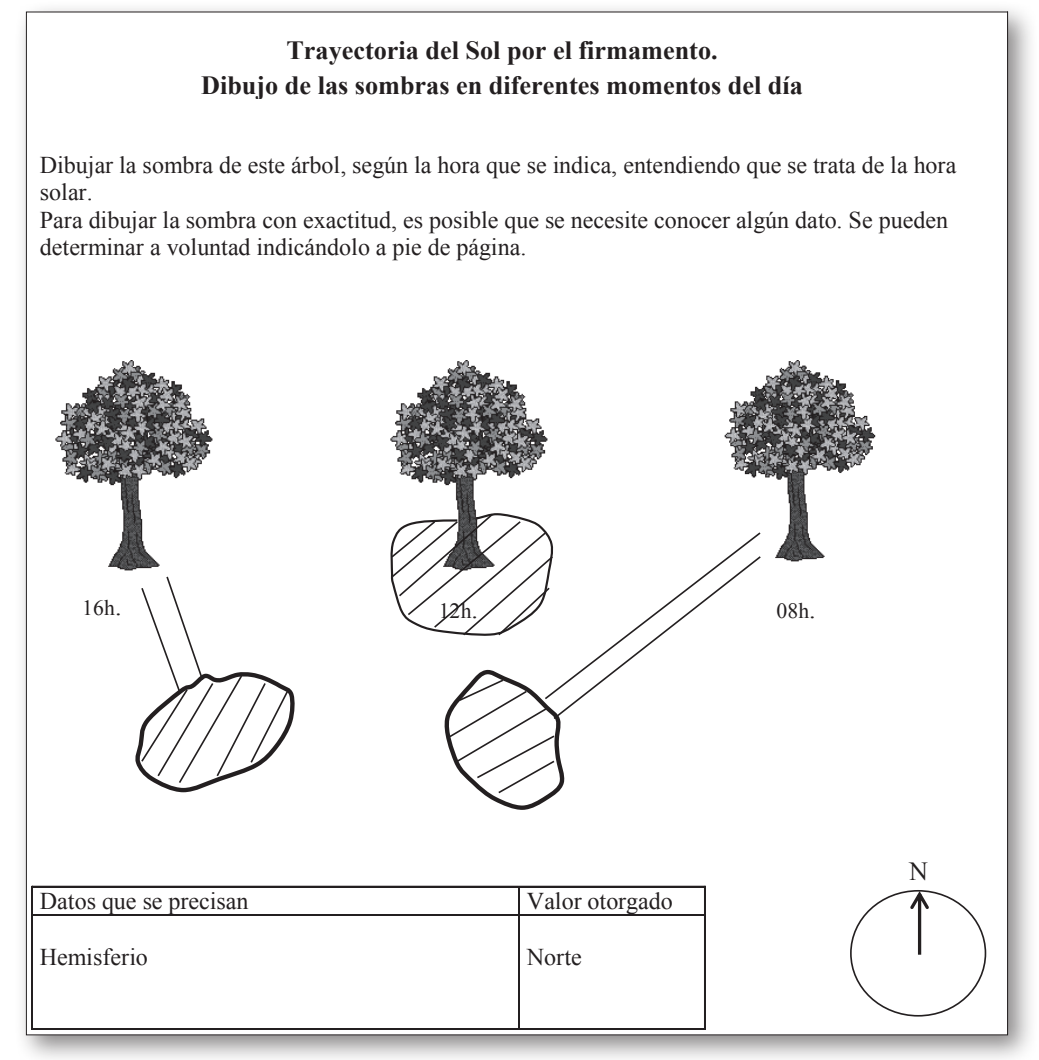

Fig. 1. Ideas del alumnado sobre la sombra que arroja un árbol en diferentes momentos del día.

-Se dibuja la sombra del árbol a las $12 \mathrm{~h}$ con un círculo que rodea su base, en la que su centro coincide prácticamente con el centro de la base del tronco del árbol. Se sitúa la sombra como si el Sol estuviera en una posición perpendicular encima del árbol. Ello puede ser debido al hecho de que, tanto en la mayoría de los libros de texto como en nuestras expresiones cotidianas, señalamos que el Sol al mediodía está «en lo más alto», «encima de nuestras cabezas», "perpendicular sobre los objetos que ilumina».

-Las sombras que se dibujan de las $8 \mathrm{~h}$ y de las $16 \mathrm{~h}$ poseen una orientación aproximadamente adecuada, pero independientes una de la otra. No se dibujan simétricas aun cuando el tiempo transcurrido por la mañana y por la tarde es el mismo (4 horas) tomando como referencia las $12 \mathrm{~h}$ del mediodía. En la ficha de la actividad se indica que estamos tratando horas de sol, no de reloj que cambian por 
motivos económico-sociales, por lo tanto las sombras que se proyectan 4 horas antes y 4 horas después del cénit son aproximadamente las mismas pero simétricas.

-En muchos casos, las sombras se dibujan sin que partan del árbol. Es decir, aparecen como flotando sin definición de su arranque. Esta situación es la expresión gráfica del hecho de que las ideas previas se manifiestan muchas veces de forma difusa, poco concreta y con un lenguaje impreciso (Driver, 1986). A muchos les cuesta señalar con precisión el lugar de inicio de la sombra jugando con la ambigüedad.

-El recuadro en el que se pide que concreten el valor de las variables que necesitan conocer para hacer el dibujo de la sombra lo más exacta posible se encuentra, de forma generalizada, en blanco. A lo sumo, muy pocos indican el hemisferio Norte donde viven. Nadie señala otra variable. Ninguna respuesta encontramos de escalas de dibujo ni de altura del Sol para hacer la representación de la sombra «lo más precisa posible».

-Coincidimos con la bibliografía en el hecho de que a medida que aumenta la edad se abandonan las ideas más sencillas, aunque ello no significa que se adquieran las nociones científicas que expliquen las observaciones. En nuestro caso, y ante el problema de la sombra del árbol, a los más pequeños no les importa dibujarla hacia el Norte, hacia el Sur, o la de una hora hacia un punto y la siguiente hacia el otro. Muchas veces lo hacen atendiendo a la existencia de "papel libre para dibujar", es decir, donde encuentran hueco. Pero en el alumnado de mayor edad aunque sus respuestas son menos azarosas no por ello coinciden con las más adecuadas.

\section{SECUENCIA DE ENSEÑANZA-APRENDIZAJE DE LAS ESTACIONES}

Hemos señalado que nuestros esfuerzos se centran en la realización de actividades dirigidas al alumnado de educación secundaria para que pueda elaborar un modelo Sol-Tierra que sirva para interpretar las observaciones astronómicas de forma científica. Entre los diversos aspectos que podemos estudiar, formas y distancias de los astros, la Luna y sus fases, el día y la noche, etc., nos dirigimos especialmente al problema de las estaciones por entender que es fundamental en la elaboración de dicho modelo.

Para ello nuestra propuesta se centra en la siguiente secuencia de actividades:

- El movimiento de la sombra que arroja un árbol (ya comentada).

- Manejo del gnomon y dibujo de la trayectoria del Sol.

- Comparación de la trayectoria en diversos días.

- Paso de la visión geocéntrica a la heliocéntrica.

- Aplicación del modelo a diferentes contextos.

\section{Manejo del gnomon y dibujo de la trayectoria del Sol}

Nuestro punto de partida se centra en las observaciones del movimiento aparente del Sol que podemos llevar a cabo con el gnomon, las cuales son muy útiles en la construcción del modelo Sol-Tierra. El gnomon es uno de los instrumentos más utilizados para llevar a cabo estas observaciones por su sencillez, economía, y por los resultados tan sorprendentes que obtenemos con él. Un simple lápiz hincado en plastilina permite comprobar que el Sol no está quieto en el cielo y son actividades que realizamos ya en la educación primaria (S. García Barros y C. Martínez Losada, 2013; Gangui, Iglesia y Quintero, 2009). Partir de las observaciones sobre el movimiento aparente del Sol, nos puede servir como esquema explicativo del modelo que tratamos de construir (Martínez Sebastiá, 2004). Con él podemos evidenciar el lugar de la salida y puesta de Sol, el movimiento de las sombras, las horas de luz, la perpendicularidad de los rayos solares, etc. 
Al trazar las sombras que proyecta el gnomon sobre el suelo en diferentes días observamos trayectorias distintas, lo que implica que el recorrido del Sol por el firmamento varía según el día de observación. Esto se concreta en que el Sol se encuentre a diferente altura sobre el horizonte a medida que transcurre el día; que alcanza distintas alturas, sale y se pone por diferentes lugares a lo largo de las estaciones, y podemos hacer un seguimiento de su trayectoria dibujando las curvas que su sombra describe.

Este estudio de las sombras que arroja un objeto durante las horas de luz, realizado de manera más o menos aproximada, resulta adecuado para obtener una idea del tránsito del Sol por el firmamento. Sin embargo, queremos profundizar en estas observaciones y operar con los resultados que obtengamos. Al solicitar una mayor concisión y exactitud en el dibujo de las sombras del árbol en distintos momentos del día, en la primera actividad que hemos propuesto, aparecen problemas a los que el alumnado no había prestado atención: la altura del árbol, la escala del dibujo, las coordenadas del lugar, etc., pero sobre todo la altura del Sol sobre el horizonte o ángulo que sus rayos forman con la Tierra, y la dirección de estos con respecto al Norte.

Nuestro propósito no es dibujar el recorrido de las sombras sino el recorrido que hace el Sol por el firmamento visto desde la Tierra. Si somos capaces de situar en un plano, definido por unas coordenadas que representan los puntos cardinales, un punto que simboliza la ubicación del Sol, habremos conseguido nuestro propósito. Si esto lo hacemos varias veces, podremos unir los puntos y trazar la trayectoria que el Sol realiza en su movimiento aparente por el firmamento, pero representado en el plano (Gavidia, 1986). Al determinar esta trayectoria, no solo conoceremos cuándo comienza y termina de iluminarnos, por dónde «sale» y se «pone» el Sol, sino que podremos averiguar la sombra que arroja cualquier objeto a una determinada hora del día.

Los datos que necesitamos para realizar la gráfica de este recorrido son los mismos que necesitábamos para trazar la sombra del árbol: la altura a la que se encuentra el Sol o ángulo que sus rayos forman con el horizonte $(\alpha)$, y la dirección de estos en relación con el Norte geográfico $(\beta)$. Estos datos los podemos obtener fácilmente con la brújula, una cinta métrica y el gnomon, del que debemos conocer su altura (a).

La forma de operar para situar el lugar que ocupa el Sol en el plano en un momento determinado es el siguiente: situamos el gnomon en un lugar soleado y medimos la longitud de su sombra (b) y, con la brújula, el ángulo de esta con respecto al Norte $(\beta)$, tal y como indica la figura 2. Para ello, solo tenemos en cuenta la visión en planta de la sombra del gnomon. Sin embargo, la altura del Sol sobre el horizonte, a la que hemos denominado ángulo $\alpha$, es una visión en alzado de la figura que contemplamos.

Si unimos el punto final de la sombra con el extremo superior del gnomon, se forma una recta (rayo solar) al final de la cual se encuentra el Sol. El ángulo que forma esta recta imaginaria con la horizontal es el ángulo $\alpha$ o inclinación del Sol sobre el horizonte. Lo podemos obtener a partir de su tangente, que calculamos conociendo la sombra que arroja el gnomon (b), y su altura (a): $\tan \alpha=\mathrm{a} / \mathrm{b}$. 


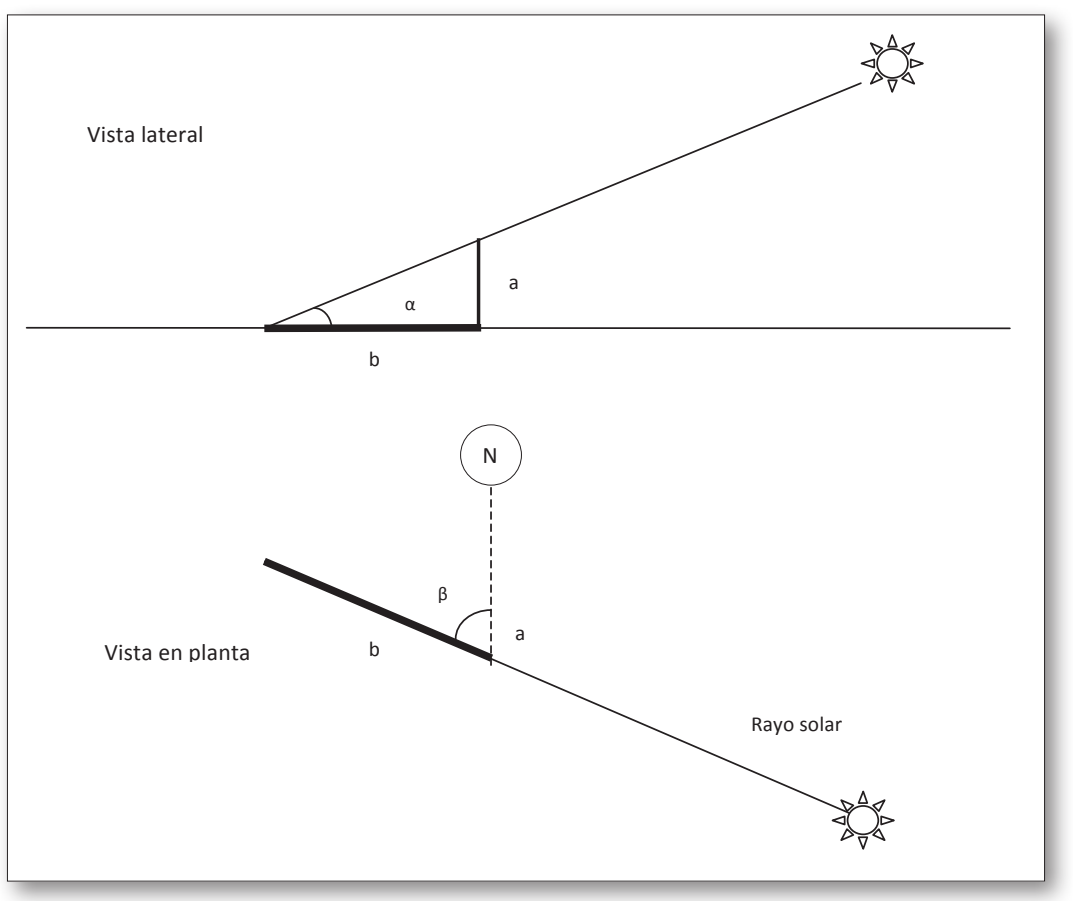

Fig. 2. Cálculo de la altura del Sol sobre el horizonte a través de la sombra del gnomon.

Con estos datos podemos situar en el plano el punto que representa dónde se encuentra el Sol en el firmamento en el momento de la medición. Actuaremos de la forma que indica la figura 3:

a) En un papel dibujamos una circunferencia que simboliza el límite de la bóveda celeste. Nosotros, como observadores, nos encontramos en el centro de esta. También en el centro de este círculo se encuentra el gnomon. Señalamos la posición Norte.

b) Dibujamos en el centro de la circunferencia el ángulo $\beta$ que forma la sombra del gnomon con el Norte. Señalamos la hora de lectura, en este caso las $10 \mathrm{~h}$.

c) Indicamos la dirección en la que se encuentra el Sol en ese momento y que es la prolongación de la sombra, es decir, el rayo solar horario.

d) Ubicamos la posición del Sol en su rayo solar horario, para lo que debemos tener en cuenta el ángulo $\alpha$ y realizar las siguientes operaciones: situamos en el centro de la circunferencia el ángulo $\alpha$ con un transportador de ángulos tomando como base el rayo solar horario. Trazamos el lado correspondiente que delimita el ángulo $\alpha$, y en el punto de intersección con la circunferencia bajamos una perpendicular al rayo solar horario. Su confluencia nos indicará la situación del Sol en el firmamento en ese momento. El resultado es independiente de hacia dónde se sitúe el ángulo $\alpha$, a la derecha o izquierda del rayo solar. 


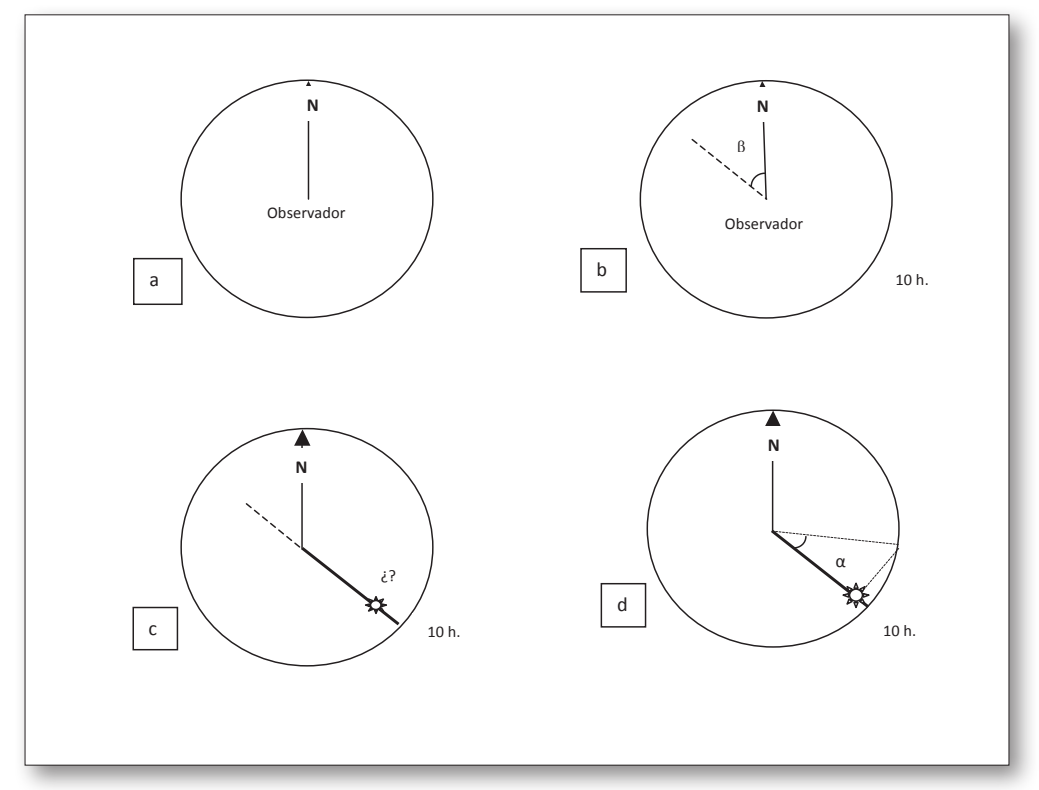

Fig. 3. Situando la posición del Sol de las $10 \mathrm{~h}$ sobre un plano.

Repitiendo este mismo procedimiento unas cuantas veces obtenemos varios puntos y, al unirlos, trazamos la trayectoria que el Sol sigue por el firmamento. Hay que recordar la existencia de puntos simétricos en esta trayectoria: los pertenecientes a horas con la misma diferencia de tiempo con respecto a las 12, es decir, la sombra de las 11 y de las 13 será aproximadamente la misma, como también la de las 9 y la de las 15. Con la trayectoria del Sol dibujada en el plano conocemos en todo momento su posición con respecto al Norte y su inclinación sobre el horizonte y, por tanto, las longitudes y direcciones que arrojan las sombras de los diferentes objetos de los que conozcamos su altura. Los puntos tangentes de esta trayectoria con el círculo, la salida y puesta del Sol, nos lo frecen los medios de comunicación, pero hay que estar atentos a la dirección de la sombra o rayo solar. A continuación, y a modo de ejemplo, se reproduce una de estas trayectorias del Sol (fig. 4).

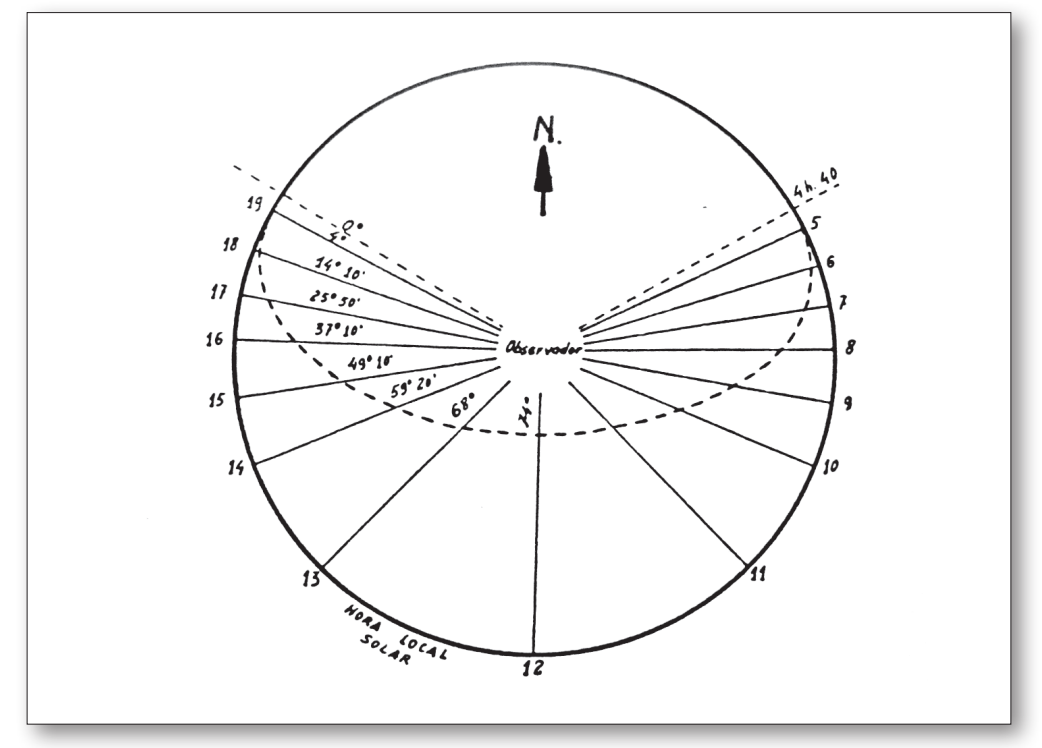

Fig. 4. Trayectoria del Sol sobre la ciudad de Valencia el día 21 de junio. 


\section{Comparación de la trayectoria en diversos días}

Las estaciones, con sus cambios de temperaturas, diferentes horas de luz solar, distinta inclinación de los rayos solares, distintos lugares de «salida» y «puesta» del Sol por el horizonte, etc. pueden ser estudiadas con facilidad observando las gráficas de sus trayectorias. Para ello podemos presentar al alumnado preguntas como las siguientes, que les permitan explicarse los diferentes fenómenos que ocurren durante las estaciones.

*Las trayectorias que realiza el Sol en el firmamento en las distintas épocas del año ¿son las mismas?

¿En qué se diferencian? ¿Cuál es su consecuencia?

En las trayectorias dibujadas de distintos días del año que se presentan (fig. 5 a, b, c y d), podemos observar fundamentalmente lo siguiente:

En invierno las trayectorias son más cortas, esto se debe a que el tiempo que transcurre el Sol por el firmamento es menor; en el lugar donde nos encontramos (Valencia, España), la salida no corresponde con el Este sino con el Este-Sudeste, y la puesta tampoco con el Oeste, pues está desplazada hacia el Sur; la trayectoria transcurre alejada del centro, lugar donde nos encontramos, lo que indica que el Sol posee una mayor inclinación y por tanto las sombras serán más alargadas. A mediodía las sombras se alejan de la base del objeto hacia el Norte. Todo ello explica que en invierno los días sean cortos y la temperatura fría en lugares situados al Norte del trópico de Cáncer.

En estos mismos lugares, podemos observar que a medida que se acerca el verano: las trayectorias son más largas, esto es, el tiempo que transcurre el Sol por el firmamento va aumentando; la salida del Sol se acerca más al Este y lo sobrepasa hacia el Norte; la puesta del Sol se acerca al Oeste y también lo sobrepasa hacia el Norte; la trayectoria se acerca cada más del centro, lo que indica que el Sol posee una menor inclinación, cada vez más perpendicular y por tanto las sombras serán más cortas. A mediodía las sombras se aproximan a la base del objeto. Estas observaciones, ilustradas sobre el papel, explican que en verano los días sean más largos, las temperaturas aumenten y las sombras del mediodía sean más cortas.

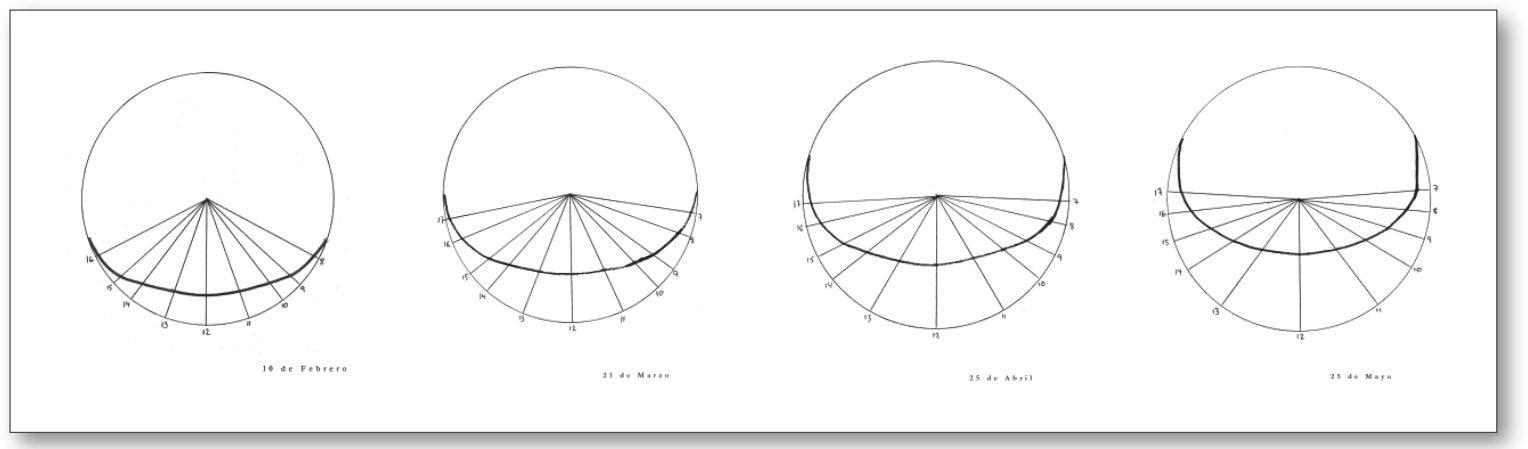

Fig. 5. a, b, c y d. Trayectoria del Sol por el firmamento en diversas épocas del año.

\section{Paso de la visión geocéntrica a la heliocéntrica}

Todas las observaciones realizadas hasta el momento se han llevado a cabo desde la Tierra, lo que podría dar lugar a mantener una visión geocéntrica. Necesitamos cambiar nuestro punto de referencia y tratar de interpretar los datos que conocemos desde otra perspectiva. Para ello sugerimos la realización de una actividad como la que se expone en la figura 6, en la que tratamos de ubicar las diferentes trayectorias dibujadas del movimiento aparente del Sol con las distintas ubicaciones de la Tierra a lo largo de su trayectoria elíptica. 


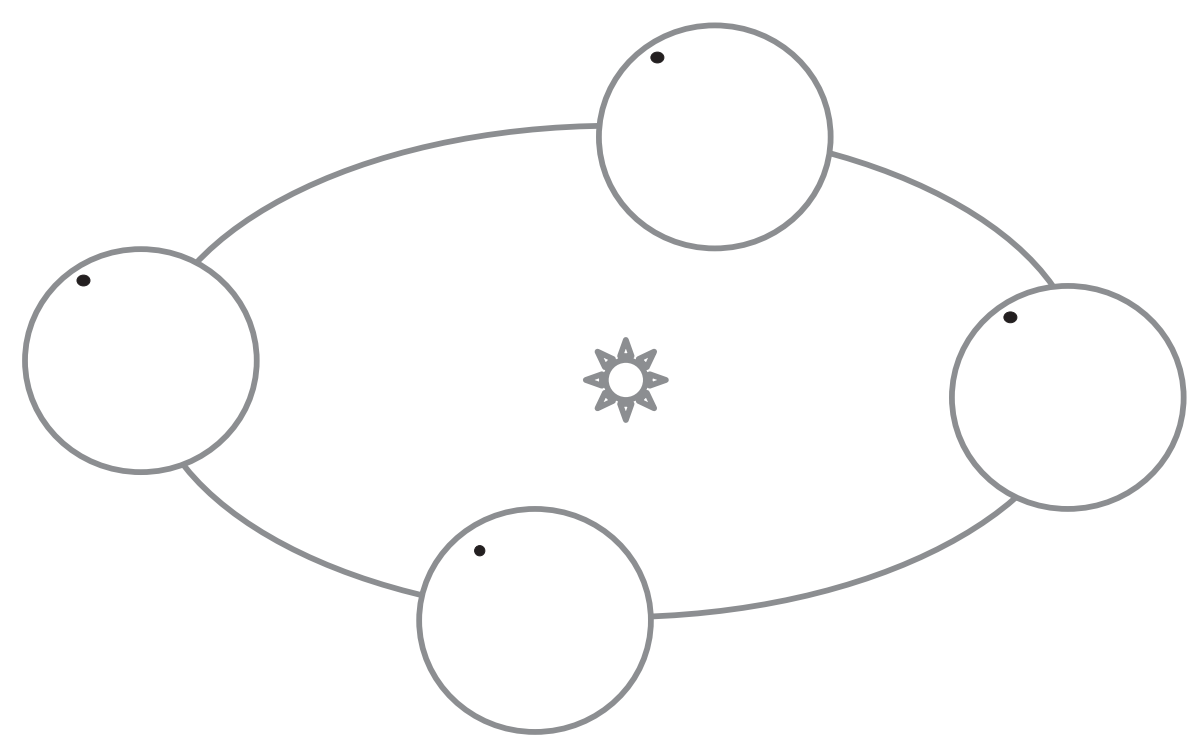

- Dibujad el eje de rotación de la Tierra en las 4 representaciones que aparecen en la figura.

- Señalad la zona iluminada del planeta en cada una de las 4 situaciones.

- Trazad el recorrido que realiza el punto señalado en el planeta debido al movimiento de rotación.

- Dibujad, al lado de cada una de las siluetas del planeta, la trayectoria del Sol que vería un observador situado en el punto indicado que se encuentra en el hemisferio Norte.

- Haced lo propio pero con un punto elegido en el hemisferio Sur.

Fig. 6. Sistema Sol-Tierra.

\section{APLICACIONES DEL MODELO CONSTRUIDO ¿QUÉ HEMOS OBTENIDO Y QUÉ PODEMOS HACER CON ELLO?}

Hemos conseguido representar la trayectoria del Sol por el firmamento en un plano, limitado por un círculo que representa la bóveda celeste o bien el espacio de horizonte y firmamento que abarca nuestra mirada. Esta gráfica tiene la particularidad de ofrecernos la trayectoria que sigue el Sol y, por tanto, su situación en todo momento, su inclinación sobre el horizonte y la posición con respecto al Norte. A partir de estos datos podemos plantear actividades que permitan progresar en la construcción del modelo Sol-Tierra, aplicar este modelo a diversas situaciones y comprobar su utilidad.

Las actividades que se presentan a continuación son ofrecidas al alumnado de primer curso de bachillerato en la asignatura de Ciencias para el mundo contemporáneo, 16-17 años.

\section{Avances en la construcción del modelo Sol-Tierra. Las observaciones de Galileo}

La comprensión del sistema Tierra-Sol es uno de los elementos clave en la historia de la humanidad, en la evolución de las ideas y en el desarrollo científico. Jones, Lynch y Reesink (1987) señalan: «El mo- 
delo heliocéntrico opuesto a la descripción de la Tierra como centro del sistema solar tiene un enorme significado intelectual. La transición representa un indicador clave de las tensiones que existen entre mito, magia, ciencia y religión». Sin embargo, todas las indagaciones, mediciones y apreciaciones que hemos presentado hasta ahora las hemos hecho tomando como punto de referencia la Tierra, lugar desde donde realizamos nuestras observaciones, por lo que se puede pensar que inducen y desarrollan la idea de que nuestro planeta está fijo y es el Sol el que gira a su alrededor.

En la actividad anterior, hemos presentado el modelo heliocéntrico tratando de compaginarlo con las observaciones que tenemos que responden a la visión geocéntrica, pero ¿por qué hay que ofrecer otro punto de referencia, otro modelo? ¿Por qué esta duda? Es un momento adecuado para presentar al alumnado la dificultad de abandonar la visión geocéntrica, que explica lo que vemos desde nuestro observatorio y permite acercarnos al nuevo paradigma. Pero para ello debemos presentar pruebas y evidencias que demuestren el heliocentrismo. Ello supone conocer las observaciones de Galileo e invitar a cambiar nuestro punto de observación.

Las controversias que mantuvo Galileo con sus opositores y una aproximación al Diálogo, con los personajes Filipo Salviati, Giovan Francesco y Simplicio, es una ocasión inestimable para entender las dificultades de abandonar interpretaciones de la realidad "consagradas» y aceptar nuevas teorías que significan una nueva visión del mundo. Entre los datos más importantes que Galileo presenta hacia el heliocentrismo, nos podemos servir de los satélites de Júpiter (por lo que la Tierra no es el centro de todo lo que se mueve), y las fases de Venus y sus diferentes distancias en relación con la Tierra. Si unas veces se encuentra más cerca de la Tierra que el Sol y otra más lejos, los movimientos no pueden ser regulares o la Tierra no es el centro. Las diferentes visiones de Venus no pueden explicarse con la Tierra en el centro del movimiento, pero sí situando en ese punto al Sol.

Hay que señalar que estas actividades se realizan fundamentalmente con alumnado de secundaria, que ya tienen construido un esbozo del modelo Sol-Tierra y saben que la Tierra es la que gira alrededor del Sol; pero además, y como señala Lanciano (1989), es posible pensar como Copérnico y hablar y actuar como Ptolomeo. Esta autora afirma que «nadie deja de ser completamente tolemaico para hacerse simplemente copernicano. Se trata, más bien, de ser capaz y consciente de aceptar la posibilidad de mantener dos modelos diferentes, dos modelos de lenguaje para hablar de lo mismo: mantener la visión tolemaica cotidiana y su relatividad con respecto a un sistema del mundo en el que todo se mueve y no existen centros locales relativos». Hacer presente esta realidad es lo que ha tratado esta actividad.

\section{Aplicación del modelo a contextos de la vida real}

No parece necesario recalcar la importancia de aplicar lo que se estudia en el aula a la vida cotidiana. La contextualización de los temas tratados es una de las formas más importantes de alfabetización científica y de motivación del alumnado, ya que se les muestra que los estudios no son elucubraciones teóricas. Las actividades que vienen a continuación poseen este objetivo.

* Tengo un árbol en mi jardín y quiero plantar unas margaritas y unos geranios. Estas dos plantas requieren mucho sol y me pregunto ¿cuál es la distancia menor del árbol a la que las puedo situar?

Si ya tenemos dibujadas las trayectorias del Sol en los solsticios de verano e invierno como hemos indicado, y conocemos la altura del árbol y el diámetro de su copa, podemos trazar los límites a los que llegan las sombras que arroja dicho árbol sin necesidad de tomar nota en distintos momentos del día de la longitud de su sombra. La elección de lugar de plantación de margaritas y geranios es fácil y consecuente con los resultados.

Este problema puede servir para aproximarnos a diversos conceptos de geometría: al trazar la sombra del árbol en el suelo observamos que aparece una línea cónica (hipérbola), que en verano tiene un 
sentido, en invierno otra y en los dos equinoccios es una línea recta, perpendicular a la sombra del mediodía y situada entre las dos anteriores. ¿A qué puede ser debido esto?

\section{*.Qué debemos tener en cuenta al buscar una vivienda en la ciudad, si deseamos que los rayos del Sol} incidan en ella la mayor cantidad de tiempo posible?

Este problema posee las siguientes características: a) presenta una complejidad variable y, dependiendo del nivel de su tratamiento, puede resultar sencillo o más complejo; $b$ ) tiene un carácter transversal e interdisciplinar, dado que son varias las disciplinas implicadas en él; $c$ ) conlleva problemas derivados, y d) su tratamiento en el aula contribuye a formar una conciencia sobre la problemática ambiental, sanitaria y consumerista.

Entre los contenidos que en este problema se pueden contemplar citamos los siguientes: $a$ ) conceptuales: los movimientos de la Tierra; la trayectoria del Sol por el firmamento; la inclinación del Sol en relación con su latitud y estación del año, el Norte magnético y el geográfico, etc.; $b$ ) procedimentales: sistematización en la observación y toma de datos; utilización de la brújula; cálculo de la altura de objetos inaccesibles; dibujo de planos con proyección de alzado, planta y obtención de sombras; presentación de alegaciones en los plazos de exposición pública de los planes de urbanización; reclamaciones ante la situación de que nos quiten el Sol de nuestra vivienda; etc., y c) actitudinales: ¿cómo nos gustaría que fueran las calles de nuestra ciudad atendiendo a su planificación, dirección, trazado, altura de los edificios, servicios, etc.? Una primera aproximación a los rayos solares que entran en la vivienda de un alumno de educación secundaria la podemos observar en las figuras 7 a y b.

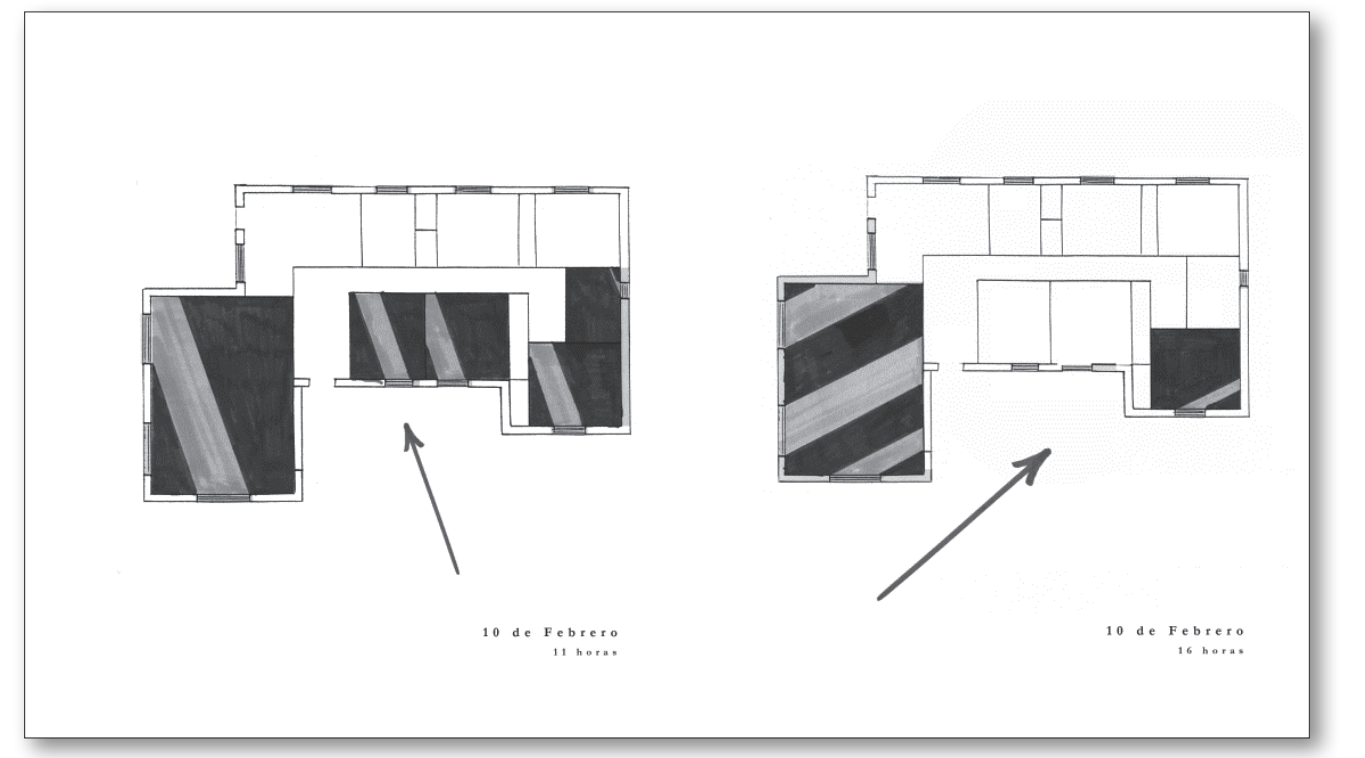

Fig. 7a y b. Iluminación de una vivienda en distintos momentos del día.

\section{* ¿Es adecuada la relación entre la altura de una calle y su anchura teniendo en cuenta su orientación?}

La aplicación de los conocimientos que estamos adquiriendo puede dirigirse al análisis de temas sociales como los relativos a la ciudad donde vivimos. De todos es conocida la transformación especulativa que están sufriendo muchas ciudades que, manteniendo el mismo trazado de las calles así como su anchura, los edificios que en ellas se encuentran son cada vez más altos. Esto, además de tener importancia en problemas de aparcamiento de coches, circulación, servicios comunitarios, calidad de vida, aglomeración de personas, etc. influye directamente en la iluminación de las calles, en el aspecto 
de la ciudad, en los rayos de sol que inciden en las viviendas y en la cantidad de luz que presentan las propias calles y casas. Estas opiniones las podemos argumentar en función de los datos que podemos obtener a partir del conocimiento de las trayectorias del Sol, como se observa en la figura 8 , en la que aparecen los cálculos de las sombras de los edificios en diferentes momentos del día y algunas fotografías que demuestran su veracidad, como también los alumnos que los hacen.

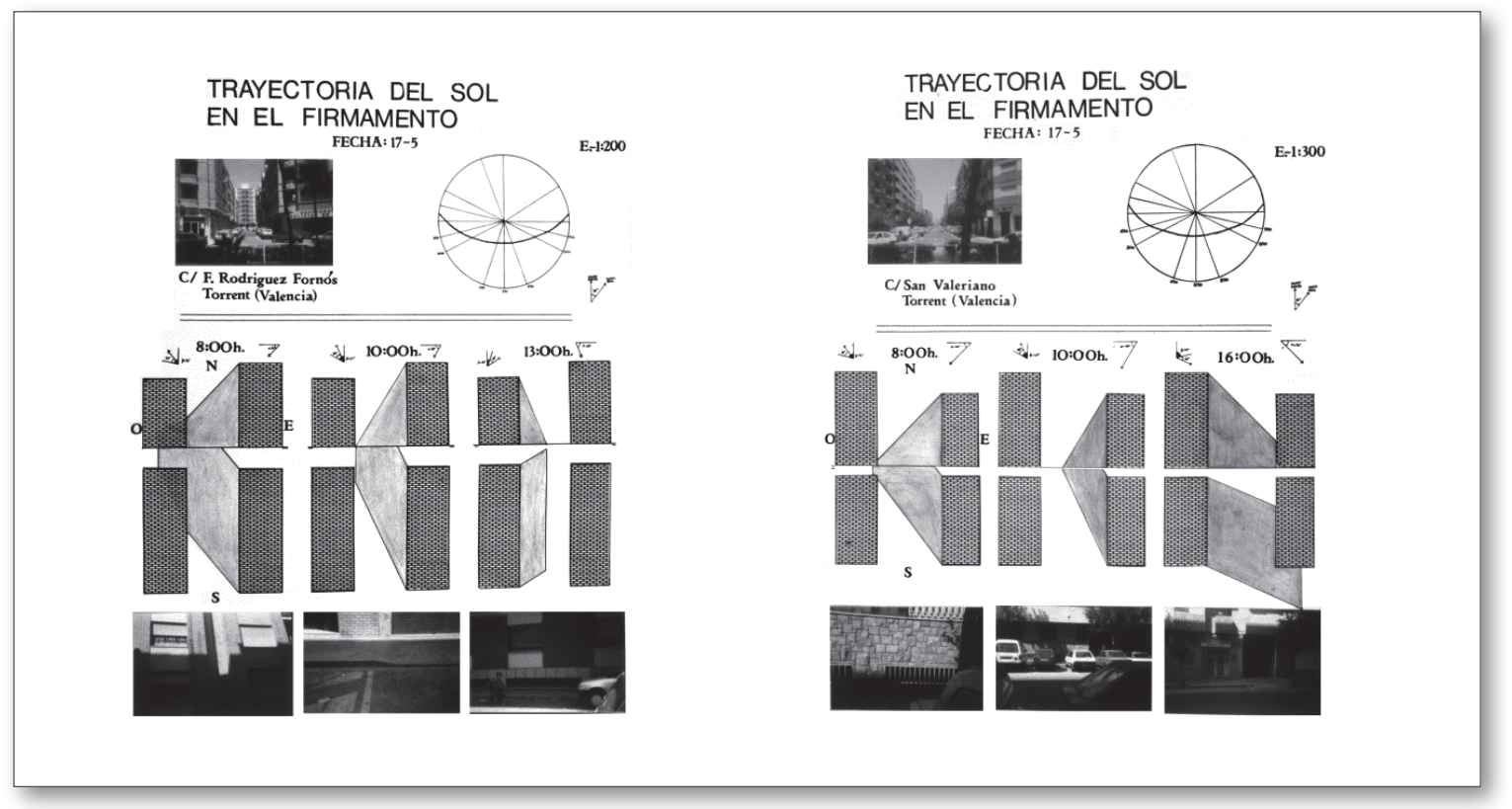

Fig. 8. Iluminación de dos calles de la ciudad de Torrent (Valencia).

\section{DISCUSIÓN Y CONCLUSIONES}

La utilización del gnomon en las observaciones astronómicas nos parece fundamental, especialmente si queremos estudiar la variación de las horas de luz y de temperatura a lo largo de las estaciones, punto de partida para una profundización mayor en el modelo Sol-Tierra. Algunas autoras (Lanciano, 1989) señalan que no es conveniente empezar con estas observaciones utilizando el gnomon, ya que ello significa dar la espalda al Sol y situar en dos dimensiones un fenómeno que ocurre en tres. Sin embargo, aparte de la dificultad que significa dar la cara al Sol y observarlo directamente, el estudio de las sombras deja constancia del movimiento aparente del Sol y permite obtener datos con los que operar dichas observaciones. Su empleo y estudio resulta adecuado para que el alumnado comprenda el funcionamiento del modelo Sol-Tierra, lo consolide, lo amplíe y lo aplique a situaciones varias.

Para complementar las observaciones con el gnomon, existen planisferios, maquetas, esferas de poliestireno, sobre las que se puede trazar la trayectoria del Sol por el firmamento (Grup Astre, 1998; Menargues et al., 2013). Son fáciles de utilizar y muy intuitivas, de forma que se adquieren con facilidad conceptos importantes como son las variaciones que se dan en las estaciones. Gangui, Iglesias y Quinteros (2009) llevan a cabo una secuencia de enseñanza en la que primero utilizan el gnomon y hacen preguntas como esta: ¿a qué se debe que las sombras no sean las mismas y cambien? Y apuntan la conveniencia de conocer la posición del Sol, la orientación de la sombra en distintos momentos del día y llevar a cabo registros a lo largo del año. Después pasan a diseñar y construir una maqueta con alambres que representen el movimiento aparente del Sol y con ello justifican el ciclo día-noche en las diferentes estaciones del año. 
Aun fijándonos en las mismas observaciones, nuestra propuesta es distinta y complementaria, pues hemos proyectado en un plano las trayectorias que observamos del Sol y que se representan de forma tridimensional en las maquetas. De este modo podemos operar con mayor facilidad a partir de las diversas «rutas», compararlas entre ellas y utilizarlas en variados problemas.

Existen autores como Parker y Heywood (1998) que indican que el alumnado presenta problemas con la interpretación de diagramas explicativos de lo que sucede, pues en el caso de las estaciones no reconoce la importancia de mantener el eje de la Tierra en un ángulo constante al plano elíptico y, en general, parece poco adecuado expresar en un plano de dos dimensiones lo que ocurre en el espacio de tres dimensiones.

Sin embargo, nuestro trabajo no consiste en realizar un diagrama al uso, en el que se intenta representar el movimiento relativo de la Tierra con respecto al Sol, tratando de explicar el porqué de las estaciones, procurando dibujar una simplificación de lo que ocurre, un modelo explicativo. Nuestro trabajo no pretende esto, ya que no es el dibujo de un modelo. Es la representación gráfica de los datos obtenidos en una serie de observaciones determinadas. Es la proyección en el plano de la trayectoria que vemos que realiza el Sol en el firmamento. Son los datos necesarios operados de forma que el alumnado configure su modelo mental.

El problema continúa siendo el paso de la visión geocéntrica a la heliocéntrica, para lo cual realizamos la actividad que aparece en la figura 6 con la que terminamos la Astronomía en la ESO. Pretendemos que el alumnado realice unos esquemas semejantes a los que aparecen en la figura 9, pero en la mayoría de los casos no lo conseguimos, de forma que se convierte en una actividad para que el profesor realice las explicaciones que considere pertinentes para redondear el tema. Pero es una de las primeras actividades con las que se vuelve a retomar la Astronomía en bachillerato.

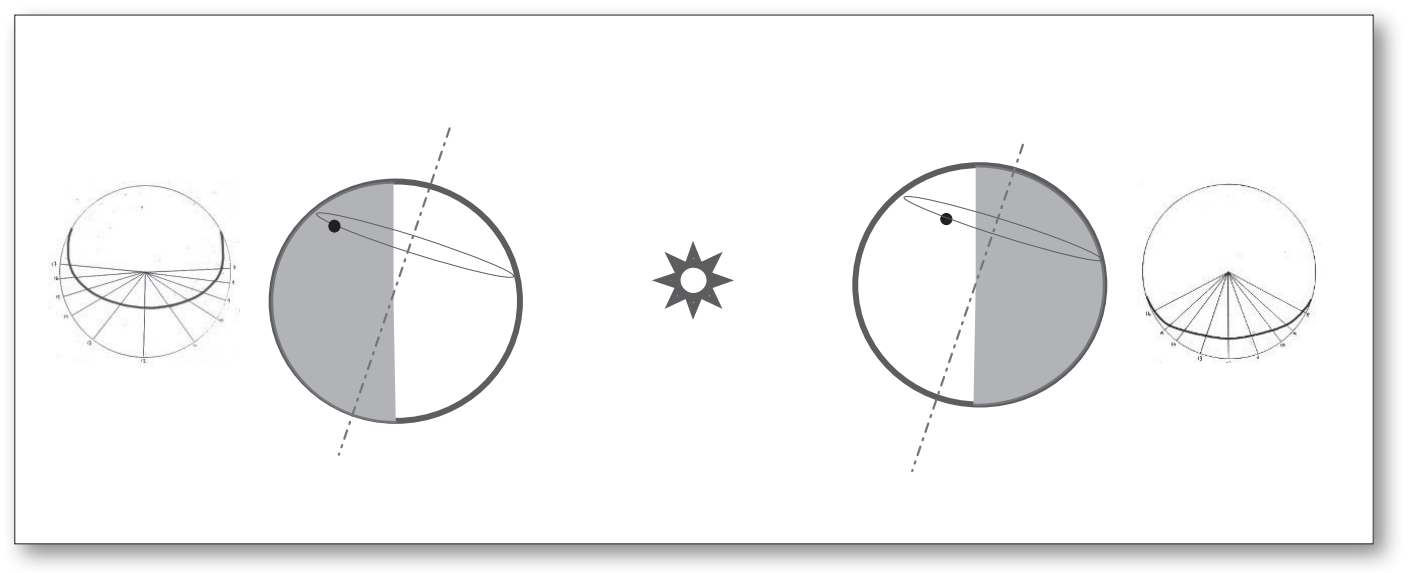

Fig. 9. Duración del día y la noche en los solsticios.

El tratamiento de este aspecto es necesario. Parker y Heywood (1998) indican que la llave que abre el rompecabezas para la comprensión del modelo es el entendimiento de las diferentes horas de luz y horas de sombra de un lugar en su movimiento de rotación diario en función de las estaciones y de dónde se encuentre. Y aunque su tratamiento no es garantía de que la mayoría del alumnado interprete satisfactoriamente todos los datos que se presentan, es estimable el avance que se consigue y es un punto de partida para estudios posteriores más exhaustivos. 


\section{REFERENCIAS BIBLIOGRÁFICAS}

Afonso, R.; Bazo, C.; López, M.; Macau, M.D. y Rodriguez Palmero, M.L. (1995). Una aproximación a las representaciones del alumnado sobre el universo. Enseñanza de las Ciencias, 13 (3), pp. 327-335.

Aтwood, R.K. у Aтwood V.A. (1997). Effects of Instruction on Preservice Elementary Teachers' Conceptions of the Causes of Niht and Day and the Seasons. Journal of Science Teacher Education, 8 (1), pp. 1-13.

http://dx.doi.org/10.1023/A:1009455201314

Bach, J. y Franch, J. (2004). La enseñanza del Sistema Sol-Tierra desde la perspectiva de las ideas previas. Enseñanza de las Ciencias de la Tierra, 12 (3), pp. 302-312.

BAXter, J. (1989). Children's understanding of familiar astronomical events. International Journal of Science Education. 11, pp. 502-513.

http://dx.doi.org/10.1080/0950069890110503

BLOwn, E.J. y BRYCE, T.G.K. (2010). Conceptual coherence revealed in multi-modal representations of astronomy knowledge. International Journal of Science Education, 32(1), pp. 31-67. http://dx.doi.org/10.1080/09500690902974207

Bryce, T.G.K. y Blown, E.J. (2012). The novice-expert continuum in astronomy knowledge. International Journal of Science Education, 34(4), pp. 545-587. http://dx.doi.org/10.1080/09500693.2011.642325

Bryce, T.G.K. y Blown, E.J. (2013). Children's Concepts of the Shape and Size of the Earth, Sun and Moon. International Journal of Science Education, 35 (3) pp. 388-446. HTTP://DX.DOI.ORG/IO.IO80/09500693.20I 2.750432

Camino, N. (1995). Ideas previas y cambio conceptual en Astronomía. Un estudio con maestros de primaria sobre el día y la noche, las estaciones y las fases de la Luna. Enseñanza de las Ciencias, 13 (1), pp. 81-96.

Cardenete, S. (2011). Sol, Tierra y Luna. Movimientos relativos y sus consecuencias. Revista Eureka sobre Enseñanza y Divulgación de las Ciencias 8, pp. 512-518.

De Manuel, J. (1995). ¿Por qué hay veranos e inviernos? Representaciones de estudiantes (12-18) y de futuros maestros sobre algunos aspectos del modelo Sol-Tierra. Enseñanza de las Ciencias, 13 (2), pp. 227-236.

Domenech, A. y Bella, T. (2000). Astronomía en la educación secundaria obligatoria. Alambique, 23, pp. 73-82.

Domenech, A.; Domenech, M.T.; Casasus, M.E. y Bella, T. (1985). Apuntes para una programación didáctica de la astronomía en la Enseñanza Media. Enseñanza de las Ciencias, 3 (3), pp. 204-208.

Dove, J. (2002). Does the man in the moon ever sleep? An analysis of student answers about simple astronomical events: A case study. International Journal of Science Education, 24 (8), pp. 823-834. http://dx.doi.org/10.1080/09500690110066935

Driver, R. 1986. Psicología cognoscitiva y esquemas conceptuales de los alumnos. Enseñanza de las ciencias, 4 (1), pp. 3-15.

Gangui, A.; Iglesias, M. y Quintero, C. (2009). Ciencias en el Aula El movimiento de las sombras. Ciencia Hoy. Instituto de Astronomía y Física del Espacio IAFE (CONICET-UBA), Argentina. Vol.19, número 110. Disponible en línea: <http://cms.iafe.uba.ar/gangui/difusion/ch/ch110/gangui-etal-ch110-prop-pedagogica.pdf>.

García Barros, S. y Martínez Losada, C. (2013) Inmersos en el aire miramos el cielo. Graó: Barcelona. 
García Barros, S.; Mondelo Alonso, M. y Martínez Losada, C. (1996). La astronomía en la formación de profesores. Alambique, 10.

Gavidia, V. (1986). Medio Ambiente y Adaptaciones. Madrid: Ministerio de Educación y Ciencia. Breviarios de Educación.

Grup Astre (1998). Materials didàctics per a l'ensenyament de l'Astronomia. Valencia: Nau Llibres.

Iglesias, M.; Quinteros, C y Gangui, A. (2007). Astronomía en la escuela: situación actual y perspectivas futuras. Actas de la XV Reunión Nacional de Educación en la Física, pp. 68-80. Disponible en línea: <http://arxiv.org/ftp/arxiv/papers/0807/0807.0418.pdf>.

Jones, B.; Linch, P. y Reesink, C. (1987) Children's conceptions of the Earth, Sun and Moon. International Journal of Science Education, 9 (1), pp. 43-53.

http://dx.doi.org/10.1080/0950069870090106

Klein, C.A. (1982). Children's concepts of the Earth and the Sun: A Cross Cultural Study. Science Education, 65 (1), pp. 95-107.

http://dx.doi.org/10.1002/sce.3730660112

KLein, J.L. y Riskin, A. (2003). Learning by the Sun: Observing Seasonal Declination With a Vertical Sundial. Journal of Science Education and Technology, 1 (1), pp. 81-88. http://dx.doi.org/10.1023/A:1022115829592

Lanciano, N. (1989). Ver y hablar como Ptolomeo y pensar como Copérnico. Enseñanza de las Ciencias, 7 (2), pp. 173-182.

López-Gay, R.; Jiménez, R.; Osuna, L. y Martínez-Torregrosa, J. (2009). El aprendizaje del modelo Sol-Tierra. Una oportunidad para la formación de los maestros. Alambique, 61, pp. 27-37.

Martínez Sebastiá, B. (2004). La enseñanza/aprendizaje del modelo Sol-Tierra: Análisis de la situación actual y propuesta de mejora para la formación de los futuros profesores de primaria. Revista Latinoamericana de Educaçao em Astronomia. RELEA, 1, pp. 7-32.

Menargues, A.; Limiñana, R.; Colomer, R. y Martínez-Torregrosa, J. (2013). Efecto de la Enseñanza Problematizada de la Astronomía diurna (ciclos y simetrías del movimiento del Sol y el modelo Sol/Tierra) en los conocimientos de los futuros maestros de primaria. Actas del XVI Congreso Nacional / II Internacional. Modelos de Investigación Educativa. AIDIPE. Disponible en línea: $<$ http://www.uv.es/aidipe/congresos/actas_XVI_congreso.pdf>.

Moreno, M. (1997). Secuenciación de contenidos y enseñanza de la Astronomía «La Tierra en el Universo». Alambique, 14, pp. 61-71.

Navarrete, A. (1998). Una experiencia de aprendizaje sobre los movimientos del sistema Sol/Tierra/ Luna en el contexto de la formación de maestros. Investigación en la Escuela, 34.

Parker, J. y Heywood, D. (1998) The earth and beyond: developing primary teachers' understanding of basic astronomical events. International Journal of Science Education, 20 (5), 503-520. http://dx.doi.org/10.1080/0950069980200501

Plummer, J.D. y Krajcik, J. (2010). Building a Learning Progression for Celestial Motion: Elementary Levels from an Earth-Based Perspective. Journal of Research in Science Teaching, 47 (7), pp. 768-787. http://dx.doi.org/10.1002/tea.20355

Ros, R. (2009). Abrir los ojos a la ciencia: una razón para enseñar astronomía. Alambique, 61, pp. 27-37.

Solbes, J. y Palomar, R. (2013). Dificultades en el aprendizaje de la astronomía en secundaria. Revista Brasileira de Ensino de Física, 35 (1), p. 1401.

http://dx.doi.org/10.1590/S1806-11172013000100016

VegA, A. (2001). Tenerife tiene seguro de Sol (y de Luna): Representaciones del profesorado de primaria acerca del día y la noche. Enseñanza de las Ciencias, 19 (1), pp. 31-44.

Vosniadou, S. (1991). Designing curricular for conceptual restructuring: Lessons from the study of knowledge acquisition in astronomy. Curriculum Studies, 23 (3), pp. 219-237.

http://dx.doi.org/10.1080/0022027910230302 


\title{
On the gnomon. Searching answers to problems
}

\author{
Valentín Gavidia \\ Dpto. Didáctica Ciencias Experimentales y Sociales \\ Universidad de Valencia \\ Valentin.Gavidia@uv.es
}

Daytime Astronomy is one of the most studied themes in both Primary and Secondary Education. It enables to introduce students to the exactitude in analysis and the presentation of problems and the argumentation. It also enables teachers to use the History of Science to attend to the conceptual change; it shows different views of the world and of science; it enables to study the ideas of students and teachers and to elaborate syllabus designs in order to collect the most frequent questions and issues that arise by looking at the sky.

This paper aims to present some contributions to make the idea of the Sun/Earth model easier and, therefore, to understand and properly interpret astronomical phenomena that are observable. In this paper we focus on the problem of seasons, their causes and their effects, leaving out other aspects like those related to the Moon. The proposal is mainly addressed to Secondary School students, who have grasped concepts such as the shape of the Sun, the Moon and the Earth, or the day and night explanation (Bryce and Blown, 2013), but also addressed to trainee teachers. The first activities are presented to 12/13-year-old students and the last to 15/16 year-old ones.

We set aside qualitative approximations about the movements of the Sun previously carried out, and we focus on more continuous and precise observations to deepen in the Sun/Earth model. It shows how to obtain more accurate data and how to operate with them, to provide students with a broader view about that model in a way that it can help them to solve daily problems and to apply to several personal and social contexts where sunlight intervenes.

The first step is an easy activity concerning the students' previous ideas about the movement of the Sun, the seasons and the importance of where the observation takes place. Then, the gnomon is studied and the shadows that it casts in different moments and days throughout the year. The special nature of this work is using the data collected, the angles and lengths of the shadows, not to draw the movement that the shadow of the gnomon casts during the day, but to draw the trajectory of the Sun through the firmament.

The comparison between different trajectories of the Sun in different days allows us to understand what happens in different seasons, in relation to the difference between day and night, the sunrise and the sunset in the skyline and the perpendicularity of sunrays. In other words, it facilitates the construction of the Sun/Earth model explaining observable phenomena that we can use to answer personal and social questions such as: What do we have to take into account when choosing a house to live in? Which is the optimal relation between height and width in the street where we live? Where in the garden should we place a daisy plant, which requires a lot of sun?

It could seem that the fact of making all the observations from the Earth looking to the Sun favors a geocentric view instead of promoting the heliocentric one. Now is the time to show Galileo's observations and to draw some activities that explain the diary observations of the trajectories of the Sun, with the inclination of the Earth's axis and its walk around the Sun.

In this way, the gnomon itself and taking measurements of the shadow it casts as accurately as possible, allows us to draw the apparent trajectory of the Sun through the firmament. It also enables us to do some activities to facilitate the understanding of the Sun/Earth model, and to apply our knowledge in solving social and personal problems. 
